\title{
Identifying the Quiet Areas of a Small Urban Setting: The Case of Mytilene
}

\author{
Matsinos G. Y.* , Tsaligopoulos A. and Economou C. \\ Mytilene, Lesvos, Greece \\ Received: 26/10/2015, Accepted: 30/11/2016, Available online: 14/02/2017 \\ *to whom all correspondence should be addressed: \\ e-mail: matsinos@aegean.gr
}

Acoustic Ecology Laboratory Department of the Environment, University of the Aegean, University Hill, Administration Bldg., 81100

\begin{abstract}
The aim of this study is to achieve a greater insight regarding quiet areas in agglomerations and contribute to their identification. The small urban setting of Mytilene located in the island of Lesvos (North Aegean, Greece), was the case study of this research. The need to control and manage environmental noise has led to the implementation of legislation that in many cases overlooks the acoustic perception of individuals. Due to the fact that noise management efforts along with the promotion of quietness in agglomerations, concerns primarily the residents of the city under consideration, it was essential to practically involve them in the decision making process. Based on citizen science contribution, a number of "places" were highlighted. The "places" mentioned from this procedure were checked by means of acoustic measurements, concerning the noise levels that occurred within the $24 \mathrm{~h}$ period. A novel method regarding the duration, repetition, check spot and the positioning of measurement was used, in order to calculate the day, evening and night period's noise levels ( $\left.L_{\text {den }}\right)$. A performance matrix was then created in order to compare the results, in relation to acoustical, functional and visual criteria. Furthermore, in order to evaluate all the potential Quiet Areas in pairwise comparisons, an Analytical Hierarchy Process (AHP) was implemented. The provision of quietness, as a direct ecosystem service, is a major indicator of environmental quality. Additionally, the way that city inhabitants perceive their acoustic surroundings could determine the character of the landscape along with the quality of the soundscape and define the meaning of quietness that still remains vague.
\end{abstract}

Keywords: Quiet Areas, Noise Measurements, Sound Recordings, Citizen Science, Soundwalk, Performance Matrix, Analytical Hierarchy Process

\section{Introduction}

\subsection{Quiet Areas}

Unlike many other environmental problems, public complaints regarding environmental noise have increased dramatically in recent years (Gidlöf-Gunnarsson, 2007). According to WHO (World Health Organization), about half of all European Union citizens live in areas that do not provide acoustic comfort to its residents (World Health Organization, Guidelines For Community Noise, 1999). Numerous studies have concluded that the existence of quiet public areas could protect urban dwellers from unwanted sounds (Chiesura, 2004) and the adverse health effects directly related to noise. The need to control and manage environmental noise has led to the implementation of legislation that in many cases overlooks the acoustic perception of individuals. Nevertheless, further research is needed in order to quantify and objectify quietness as an individual acoustic aspect of a city and not merely as the absence of high noise levels.

Quietness is considered to be a major aspect of a healthy soundscape and areas that provide such an amenity are essential for the wellbeing of urban dwellers and the quality of the urban environment. A quiet urban area retains an anthropocentric profile, offering an acoustic "relief" to city inhabitants from environmental noise. The acoustic perception of individuals holds an important role regarding attitudes on "healthy" urban soundscapes (Jeon, 2015). The psychoacoustic terms, "liveliness", "vibrancy" "positive or negative sound" are often used in order to describe a soundscape from a human perspective. Recent research shows that it is the quality of a sound that shapes a soundscape in a negative or a positive way and not the intensity of the sounds present (Bruce, 2014). Nevertheless, human beings are considered to be both the source and the receiver, making the efforts of noise control in an agglomeration, a tail chasing procedure.

The 2002/49/EC directive relating to the assessment and management of environmental noise, defined Quiet Areas in agglomerations as "areas delimited by the competent authority, which are not exposed to a value of a noise indicator greater than a certain value set by the Member 
State, from any noise source". The specific definition offers ample room for discussion. The appeal of delimitation, contradicts with the scope of the directive concerning a common noise assessment in urban areas. We argue that this tactic confines quietness, while simultaneously surrounds Quiet Areas with noisy environments. This paradoxical approach is amplified with the homogenization of sounds in terms of their intensity, overseeing the positive effect they may offer upon the urban soundscape users.

A rural quiet area serves different vital purposes, mainly concerning conservation policies for natural protected areas. Therefore, the issue of acoustic perception of individuals in rural quiet areas is unrelated, for the reason that human beings are considered indirect beneficiaries. A very effective way to locate rural quiet areas is to calculate the distance that noise from various sources like the road network propagates (Votsi et al., 2012). These distance based criteria, are probably not useful in urban areas due to the compact structure of most agglomerations.

\subsection{Acoustic Perception of Quietness}

Soundscapes are directly associated with the landscape and the type of land use. The need for quietness is linked to noise sensitivity and the sound perception of individuals. If urban sounds are perceived as a negative factor the need for quietness is higher, but if perceived as a positive factor, in terms of liveliness and vibrancy, that need is reduced (Booi, 2012). The spatial and temporal variability in sound perception is associated with the landscape structure that is formulated by human activities, biological processes and geophysical attitudes (Matsinos, 2008).

A method to assess the acoustic perception of individuals is the soundwalk. It is a way of understanding on how soundscapes are being perceived by their users (Davies, 2013). Along with the term soundscape, soundwalk is also originally attributed to R. Murray Schafer. A good example is the soundwalk conducted in the "positive soundscape project" (Davie, 2013) that highlighted both the negative and positive acoustic aspects of Manchester, UK. The soundwalking practice has proven to be a valuable tool for soundscape studies and could set the ground for future soundscape planning. The flexibility of its methodology allows novel inspired alterations that could serve different purposes regarding the scope of each research. The common Soundwalk practices, even though they are evolving, consist of several identical "steps". A pre designed route for the participants to follow, with structured questionnaires and stops in predefined checkpoints, are some of the common soundwalk characteristics.

The way that individuals perceive soundscapes, strongly relates with the activity of the listener on each occasion. The three states of listening, listening in search, listening in readiness and background listening (Jennings, 2013) could contribute to a better understanding, of the personal act of listening to a broader scale.

\subsection{Case Study Area}

The city of Mytilene, located in the island of Lesvos, according to the latest census (Hellenic Statistical Authority), has a population of 85.330 residents and a population density of 52, 26 per square kilometer. The specific city was chosen due to the diversity of its landscape, where urban and rural gradients coexist. Furthermore, islands and coastal cities are fragile systems with many ecological peculiarities. The rigorous human intervention on these systems, strongly affects the quality of the soundscape increasing the need for protection (Farina, 2012).

This study presents a novel approach, regarding the identification of potential quiet areas in small urban settlements. Research on areas with decreased noise levels could be a subject that is best known by the agglomeration's residents. The urban fabric of a city, meaning its structural individuality that shapes and differentiates neighborhoods physically, along with the socioeconomic inequalities that may occur, are a major reason for acoustic uniqueness in various scales. The citizen's knowledge about areas or spots that due to several structural and population density reasons remain unaffected by environmental noise, could navigate the scientific efforts towards the recognition of urban quiet areas.

All cities have areas quieter than others. City inhabitants use them in order to escape from their noisy surroundings, but in most cases there is no official documentation delimiting and protecting them. The use of local knowledge could navigate the efforts of area identification, without necessarily searching for "places" with low noise levels. This Citizen Science project, promotes participation in scientific research by members of the public mainly through observation and personal experience (Silvertown, 2009).

Citizen Science projects and Community-Based Monitoring (CBM) network programs, contribute with a vast amount of data regarding various ecological scientific goals such as animal appearances and abundance (e.g. bird watching), plant populations, fisheries, invasive species detection, climatic anomalies and environmental pollutants (Whitelaw, 2003; Cohn, 2008; Conrad, 2011; Resnik, 2015; Loss, 2015). Furthermore, these projects promote awareness and provide communities with numerous benefits regarding the Increase of environmental democracy (Conrad, 2010).

\subsection{Quiet Area Selection Criteria}

A list of criteria from numerous literature sources was used in order to address the issue of Mytilene's Quiet Areas. In order to gather information regarding the selected criteria, all the potential Quiet Areas of Mytilene were thoroughly studied. The selection criteria for the identification of quiet areas even though they are not limitative, differ between urban and rural areas due to different priorities and purposes that they serve. The dissimilarity regarding the acoustic indicators used and the limitations proposed is another example that highlights the different "audiences" on each type of quiet area. 
The day - evening - night noise level indicator ( $L_{\text {den }}$ ) is used solely for quiet areas in agglomerations and a noise threshold of $50-55 \mathrm{~dB}(\mathrm{~A})$ is proposed (European Environment Agency, 2014). In which the $L_{\text {day }}$ (12 hours) is the A-weighted long-term average sound level determined over all the day periods of a year, the Levening (4 hours) which is the A-weighted long-term average sound level determined over all the evening periods of a year and the Lnight (8 hours) which is the A-weighted long-term average sound level determined over all the night periods of a year. The Lden indicator (1) is calculated using the fallowing formula:

$$
\text { Lden }=10 \lg \frac{1}{24}\left[12 * 10 \frac{\text { Lday }}{10}+4 * 10^{5+\frac{\text { Levening }}{10}}+8 * 10^{10+\frac{\text { Lnight }}{10}}\right]
$$

Health restoration and nature protection are considered to be major aspects of both urban and rural quiet areas. As described in EEA's Good Practice Guide on Quiet Areas (European Environment Agency, 2014), people living in or near quiet areas, do not suffer the negative health effects which may occur to those exposed to the average soundpressure levels in an agglomeration. Therefore, areas that could provide a healthy soundscape, expanding from lower $\mathrm{dB}(\mathrm{A})$ levels, to decreased anthropogenic sounds, are preferable.

Urban green spaces that are directly associated with biodiversity levels, are often correlated with healthy soundscapes, creating a positive perception on its acoustic condition. Therefore, urban parks are placed amongst the top preferences of quiet area selection (Brambilla, 2013). Environmental noise in agglomerations burdens both, the quality of life and the quality of the overall urban environment. Numerous studies have concluded that the existence of urban green areas is beneficial regarding the mitigation of environmental noise and of other environmental pressures. Healthy, fully functioning urban ecosystems are more resilient to environmental pressures and therefore more flexible in terms of adaptation to climate change (Munang et al., 2011). The increased levels of biodiversity and the existence of both urban and rural Quiet Areas are interdependent (Votsi et al., 2012, European Environment Agency, 2014). The ecosystem services that urban Quiet Areas provide are amongst others (Yang et al. 2015; Tratalos et al., 2007, Fisher et al., 2009), the enhancement of the inhabitant's well-being, recreation, the mitigation and adaptation of climate change impacts, the microclimate regulation.

The visual criteria, refers to the existence of natural or cultural established values in official documents. Furthermore, recreation as an activity varies between moderate, intensive and passive in urban and rural areas. The ideal size of a quiet urban area varies between 100 $100.000 \mathrm{~m}^{2}$, while in rural quiet areas $0.1-100 \mathrm{~km}^{2}$. Furthermore, criteria regarding the user's acoustic perception are yet to be assessed (European Environment Agency, 2014).

The QUADMAP project (QUiet Areas Definition and Management in Action Plans) conveyed methods and selection criteria regarding the identification, delineation, characterization, improvement and management Quiet
Areas in agglomerations as defined in the Environmental Noise Directive 2002/49 (Aspuru et al., 2016). The methodology proposed consists of three stages. The first stage involves procedures regarding the emergence of the potential quiet areas. The second stage, concerns the analysis of these areas regarding on their acoustic or nonacoustic characteristics similar to safety and cleanliness. The third stage regards the management of the potential Quiet Areas regarding the preferable conditions, in order to promote low noise levels, or recreation opportunities and other social activities.

Finally, an additional approach that could be used in order to assess potential Quiet Areas is the use of acoustic indices, resulted from field soundscape recordings. The soundscape index NDSI (Normalized Difference Soundscape Index), assesses the level of human acoustic disturbance, by calculating the analogy of biophony and anthropophony in a soundscape (Fuller et al., 2015). The indices' aim is the evaluation of the anthropogenic disturbance on a soundscape, utilizing the fact that the man made sounds range between $1-2 \mathrm{kHz}$, while biological sounds range between $2-8 \mathrm{kHz}$.

The calculation of the specific index (2) is based on the following association:

$$
\text { NDSI }=\frac{\text { biophony-anthropophony }}{\text { biophony+anthropophony }}
$$

Furthermore, the index has a range in scale from -1 to 1 , with 1 indicating that an acoustic signal contains only biological sounds (Kasten et al. 2012; Fuller et al., 2015).

\section{Methodology}

In order to highlight the possible Quiet Areas of Mytilene, a protocol was designed that consists of 8 phases (Fig.1). In order for this protocol to be applicable to other cities it was deemed necessary to practically involve residents to the procedure. Using citizen science techniques the potential Quiet Areas of Mytilene were highlighted. A list of criteria was used in order to assess the alternative Quiet Areas of Mytilene. A series of measurements and recordings were conducted using strict common protocols, in order to collect data about the areas under consideration. With the aim of prioritizing the mentioned possible Quiet Areas an Analytical Hierarchy Process (AHP) tool was used. Soundwalks are proposed to be conducted to the resulted area in order to assess the way that is being perceived by its users. Finally, action plans should be designed and implemented.

\subsection{Citizen Science Contribution - Interviews}

Acoustically themed sociological surveys could provide with valuable data for numerous purposes. Nevertheless, the differentiation in their objectives fails to give the opportunity for comparison on the acoustic quality of a city, or the levels of exposure of its residents, with those of another city (Brown, 1987). The use of local knowledge aided the identification of the areas that are perceived as quiet. A total of 55 members of the academic community permanently inhabiting the city of Mytilene, were asked in advance to monitor their daily routine by mainly observing 
their acoustic surroundings. The next step was a follow up interview for each individual with the help of a semi structured questionnaire. The purpose of this procedure was to highlight the areas perceived as quiet in order to incorporate them in the measurement process. Furthermore, their sensitivity to noise at city, neighborhood and home level was discussed.

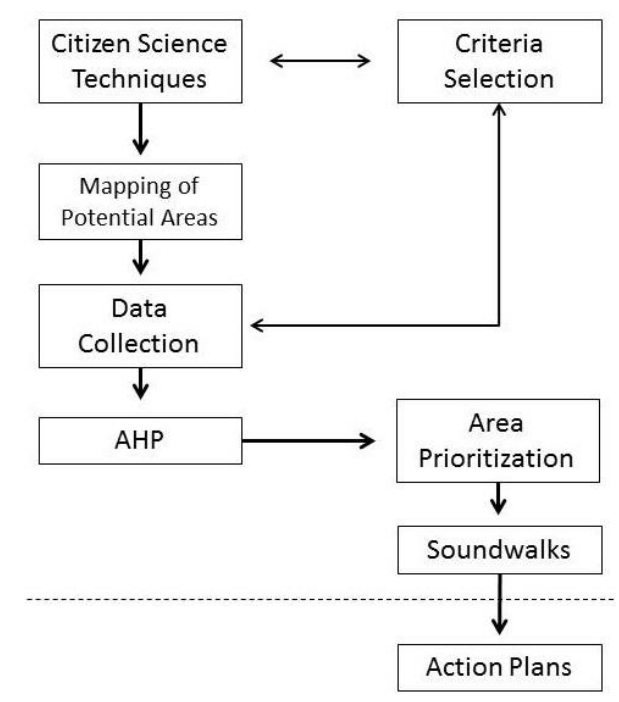

Figure 1. Quiet Area Identification Flow Chart

\subsection{Measurement areas and check spots}

Urban settlements have been spatially divided into districts and neighborhoods since the distant past. These places or zones retain social, economic and spatial significance hence creating different urban environments (Smith 2010, Sharifi 2015) and soundscapes. A place can be defined as "a small, three-dimensional urban space that is cherished by the people who inhabit it for all that it represents or means to them" (Friedmann, 2010). That cherished uniqueness could also be attributed to the specific soundmarks that shape the character of each neighborhood.

In order to obtain a realistic outcome regarding Mytilene's acoustic existing condition, a scaling down system was proposed, from city, to neighborhood, to check spot. The city of Mytilene was segregated in 11 neighborhoods (the city Centre, Sinikismos, Epano Skala, Kastro, Kioski, Limani, Chrisomallousa, Kallithea, the Stadium area, Sourada and the local University area). These neighborhoods differ both acoustically and visually, while most of them include, urban green spaces, parks, hospitals, schools and archeological sites.

All measurements and recordings were conducted using the same protocol during the spring and summer season of 2012 (May 1st - July 30th). According to the European directive 2002/49 relating to the assessment and management of environmental noise, the day period lasts 12 hours, the evening period 4 hours, and the night period 8 hours. In order to obtain a realistic result regarding $L_{d e n}$, three measurements for each period were conducted (Table 1).
The mean of the measurements for each period was calculated in order to obtain a single number that represented the period's equivalent continuous sound level ( $L_{\text {Aeq }}$ ). The outcome was then integrated in the $L_{\text {den }}$ formula, after the necessary adjustments needed for the calculation. In order to predict unexpected sonic events, the 2002/49/EC directive proposes a $5 \mathrm{~dB}(\mathrm{~A})$ penalty added for the evening period and a $10 \mathrm{~dB}(\mathrm{~A})$ penalty added for the night period.

Table 1. Measurement Time Schedule

\begin{tabular}{llll}
\hline Measurement Protocol & \multicolumn{3}{c}{ Time of Measurement } \\
\hline$L_{\text {den }}$ & $09: 00$ & $13: 00$ & $17: 00$ \\
\hline$L_{\text {day }}(07: 00-19: 00)$ & $20: 00$ & $21: 00$ & $22: 00$ \\
\hline$L_{\text {evening }}(19: 00-23: 00)$ & $24: 00$ & $02: 00$ & $06: 00$ \\
\hline$L_{\text {night }}(23: 00-07: 00)$ &
\end{tabular}

The exact spot of each sound measurement and recording was chosen regarding the topography and the urban structure of each area. The most preferable spots, considering that they were available, were open spaces far from high walls, or sharp urban structures. The notion was to keep the measurements unaffected as much as possible, from factors like sound reflection, refraction and diffraction. Strategic positioning during the measurement, considering the size and the topography of the area, in combination with consistency to the aforementioned protocol, could result to a realistic outcome. Finally, each 10 minute sampling was carried out at a height of 1,5 meters above the ground.

The Pro-DX Vocis Castle Group integrating averaging sound level meter was used in order to collect data regarding the day, evening and night equivalent continuous sound level. Furthermore, using the same protocol, sound recordings were conducted using the Tascam DR-2d Portable digital high-resolution recorder. The sound files collected were processed in order to produce spectrograms and determine the Normalized Difference Soundscape Index (NDSI) using the $\mathrm{R}$ statistics v. 3.1.3 software (http://www.R-project.org) and the associate packages Seewave, TuneR, Ineq and Soundecology (Sueur et al., 2008; Ligges et al., 2013; Zeileis 2014, Villanueva-Rivera et al., 2015)

\subsection{Mapping}

The 2002/49 directive on the management and assessment of environmental noise has given the necessary definitions that highlight the importance of strategic noise mapping. A strategic noise map could pose as a visual aid regarding all noise sources that shape an area, in order for strategic action plans to take place. Specific noise source identification (e.g. road traffic noise) and the visualization of their propagation using various noise modeling software could be a stand-alone noise map.

In order to pave the way the city's strategic noise mapping, it was decided to segregate Mytilene on neighborhood level. All information regarding the city of Mytilene was imported to the open-source Geographic Information System software QGIS v. 2.14.7 (http://www.qgis.org) projected in the WGS84 cartographic system. The 
neighborhoods of Mytilene, the check spot areas and the potential Quiet Areas, were visualized. Finally, a coloring system was used in order to visually distinguish the neighborhoods of Mytilene.

\subsection{Quiet Area Performance Matrix and Analytical Hierarchy Process (AHP)}

By utilizing the criteria provided through the EEA's (European Environment Agency) technical report on quiet areas and QUADMAP's standards, 8 criteria were chosen in order to assess the candidate quiet areas. The (a) acoustic indicator levels $\left(L_{d e n}\right)$, the soundscape index (b) Normalized Difference Soundscape Index (NDSI), the promotion of (c) nature protection on each location, the (d) health restoration capabilities, the (e) size of each area considering it is delimited by local authorities, the opportunities for (f) recreation activities, the (g) visual established values of each area and finally its (h) use, were the selected criteria.

The alternatives were the areas proposed by Mytilene's residents that participated in the citizen science project. The 18 areas proposed were categorized in 4 different types of areas and specifically 5 Urban Green Spaces, 4 Public Spaces, 3 Archeological Sites and 6 Areas of Designated Use.

(A) 5 Urban Green Areas: (1) Mytilene's Municipal Theater, the (2) Agias Eirinis park located in the city's center, an adjacent urban green space the (3) Karapanagioti park, a smaller urban green space the (4) Epano Skala's park and the city's central (5) beach "Tsamakia".

(B) 4 Public Spaces: a small urban public space called the (6) Liberty Statue located near the city's major port, the waterfronts (7) lighthouse that serves as meeting place for Mytilene's youth, (8) Mytilene's central square which is a public space used by the citizens and the municipality of the city, for various occasions and a (9) small park located in the city's center.

(C) 3 Archeological Sites: an archeological site located in the outskirts of the city the (10) ancient theatre, the ruins of the city's (11) ancient harbor and Mytilene's Byzantine
(12) Castle which is a major archeological site with plenty of green space.

(D) 6 Areas of Designated Use: the city's major (13) walkway which also serves as part of the road network when the local market closes, a (14) school yard located in the city's center, the forecourt of Mytilene's biggest (15) church (Agios Therapontas Church), the city's (16) modern port, the General (17) Hospital area and finally the city's (18) University campus.

Following the data collection from the measurements, a performance matrix was created in order to assess each alternative individually and therefore determine the best option for a candidate Quiet Area. The alternative options (areas) were scored on a 0-1 scale, where zero is the worst - case outcome and 1 is the best - case outcome (Steele, 2009). The goal of this method was to simplify the complicated procedure of area selection, by establishing each area's overall performance.

Multicriteria Decision Making (MCDM) is a valuable tool that is used for decision making in the presence of numerus criteria. This procedure evaluates alternative options using multiple and often conflicting criteria that occasionally are difficult to quantify and measure. For this research it was decided to assess the alternative areas conjunctively, in pairwise comparisons. One of the most widely used methods for achieving this goal is the Analytical Hierarchy Process (AHP), introduced by (Saaty 2004; Saaty 2008). AHP is a method of problem decomposition into a hierarchy of sub-problems, which can be understood and evaluated better. AHP is a pairwise comparison procedure with grading scales from $1-9$, with 1 expressing the neutrality between alternatives and 9 the "extreme pro-choice". In order to assess all alternative areas highlighted by the Mytilene's residents, the Priority Estimation Tool (PriEsT) was used, which has been developed to support AHP decision making (Siraj et al., 2015). The general framework of the AHP model aided the assessment procedure (fig. 2). Experts from academia contributed to the assessment of the alternative options, using the aforementioned software. The criteria and alternative evaluation was conducted in accordance with the framework's model hierarchy, by pairwise comparison.

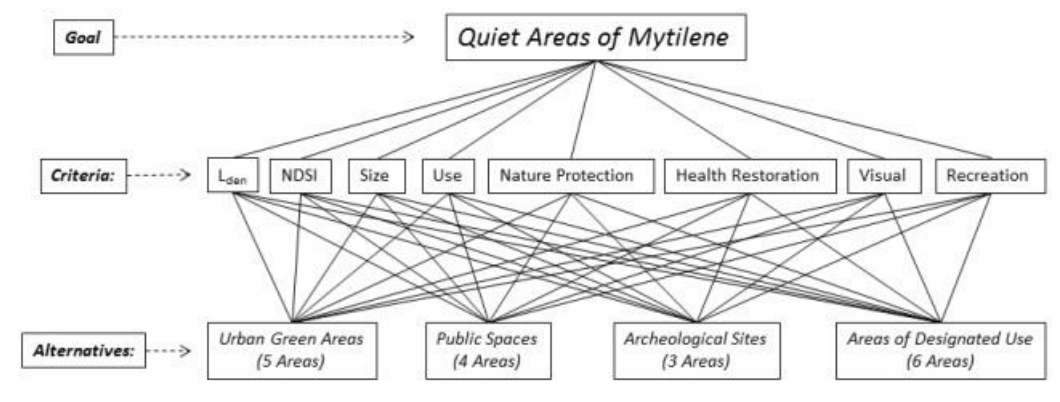

Figure 2. General Framework of the AHP Model, Regarding the Identification of Mytilene's Quiet Areas

\subsection{Soundwalks}

A very important aspect of the resulted potential quiet area is the way that is being perceived by its users. According to Hildegard Westerkamp a soundwalk is any excursion whose main purpose is listening to the environment. The purpose of this procedure is soundscape evaluation, primarily using the sense of hearing and secondary the other senses-feelings (mainly optical stimuli). Soundwalks 
are considered tools in soundscape research that could be used in order to collect the necessary qualitative and quantitative data, concerning a specific location or a larger area. The main driving force of soundwalking, is the acoustic perception of individuals while its flexible methodology, allows application in various interdisciplinary areas such as ecology, urban design and soundscape identification (Jeon et al., 2013). Furthermore, soundwalks could be used as awareness and educational tools regarding the "noise footprint" of individuals.

The researchers that undertake the implementation of a soundwalk project should consider the landscape of the location under consideration. In order to include the full audio and visual spectrum of areas with various sizes and shapes, the soundwalk stops should be chosen carefully. The stops chosen in the specific research, posed as a representation of the area's identity and purpose. In case of urban green areas, bird community structure should be taken under consideration, in order to include bird territories in the soundwalk stops. The checkpoint number depends on the size of the area and their exact location varies regarding the available landmarks and soundmarks like trees, fountains, playgrounds etc. During the first part of the soundwalk, the researcher could exploit the time given in order to collect the necessary quantitative data, by conducting noise measurements and audio recordings in every stop made by the participants.

For this research a novel soundwalk practice was suggested. It was an effort to remove the participants from a biased "mold" activity and "tune them in" to the soundscape under consideration. 5 members of the academic community contributed on the issue of acoustic perception, by participating to the organized soundwalks.
The way to familiarize the participants with the soundscape consisted of a pre designed route with 5 stops, each one with unique soundmark and landmark characteristics, in order to introduce the full spectrum of the landscape. This first part of the soundwalk was conducted as an "excursion" through the landscape, by following the rule of "silence" during the walk. The use of all senses was important for the deconstruction of the characteristics of each checkpoint, with emphasis to what could be audited. The second part of the soundwalk was a "free roam" for the participants and for the first time were introduced to a questionnaire that included open ended questions regarding on what could be audited at that specific moment and what would be the preferable sound for each location. The participants had the freedom of choice to walk through the park with no stop order or time limitation, individually or as a group. This freedom of choice contributed to the "tuning in" of the soundwalkers with their surrounding soundscape. Even though the soundwalkers were free to explore the area, all the questions concerned specific checkpoints. The specific soundwalk was conducted in the morning hours and with mild climatic conditions, avoiding strong wind and rainy days.

\section{Results and Discussion}

In terms of "noisy" as it was perceived by the residents that contributed to this research, the city level and home level were the top choices. Hence, most participants perceived their neighborhood as a "quiet" place in contrast to the other two options. The lowest score concerning quietness holds the city of Mytilene, while the wavering between noisy and quiet answers, kept a low score in the overall results (fig. 3).

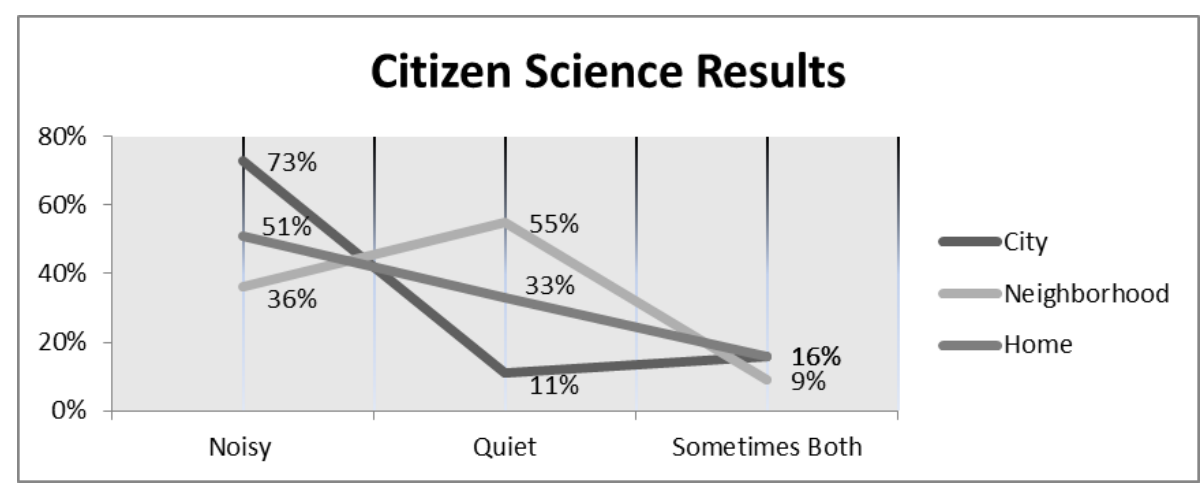

Figure 3. Citizen Science Follow-up Interview Results

The participants were asked, whether they could recall an area they use, that stands out for its acoustic quality. The areas that emerged through this question were incorporated in the measurement procedure. The harbors lighthouse was amongst the top choices made by the participants. Apart from its original purpose, the lighthouse serves as a meeting place for Mytilene's youth, due to its reasonable walking distance from the noisy streets. Nevertheless, due to its small size and lack of other important criteria, such a place could not be characterized as a "quiet area". The second most popular choice was an urban green space, located in the center of the city, the Agias Eirinis Park. The specific urban green space is a highly visited park that also serves as a recreation area. All the areas mentioned were incorporated in the measurement procedure in order to construct the acoustic profile of Mytilene (image 1), by scaling up from check spot, to neighborhood and finally to city level. The feeling of safety that was discussed during the interviews could easily be correlated with the visual isolation of an area. The $47 \%$ of 
the participants answered that they do not feel safe in the place they mentioned. Safety in an area comes with high levels of imageability, meaning a clearly understood visual environment (Luymes, 1995). The issue of safety in public spaces could be a problem concerning urban quiet areas as well.

From the statistical analysis that followed the data collection (table 2 ) it resulted that both data sets ( $L_{\text {den }}$ \& NDSI), are normally distributed. Furthermore, both data sets present a strong negative correlation (Pearson's $r=$ ,660 significant at the 0,05 level, sig.2-tailed ,003). Furthermore, an association regarding the size of the potential Quiet Areas is highlighted. The $L_{\text {den }}$ noise indicator for each area under consideration, is negatively correlated with the area's size (Pearson's $r=-, 660$ significant at the 0,01 level, sig.2 tailed,036). In contrast, the soundscape index NDSI presents a positive correlation with the area's size (Pearson's $r=, 527$ significant at the 0,01 level, sig. 2 tailed, 025). The results from the noise measurements conducted in every check spot (fig. 4) along with the spectrograms (fig. 5) and the Normalized Difference Soundscape Index results (fig. 6) could assist to a better understanding regarding the potential Quiet Area acoustic characterization. It is obvious that most of the potential Quiet Areas, apart from an archeological site (Castle) exceed the $55 \mathrm{~dB}(\mathrm{~A})$ Lden limitation.

Table 2. Measurement Descriptive Statistics. The Mean (M) and the Standard Deviation (SD) of the results

\begin{tabular}{ccccccrr}
\hline & $\mathrm{N}$ & $\mathrm{M}$ & $\mathrm{SD}$ & $\mathrm{Mdn}$ & Min & Max \\
\hline Lden & 18 & $64.7 \pm 1,61 \mathrm{~dB}(\mathrm{~A})$ & 6,8 & 65,85 & 50 & 74,8 \\
\hline NDSI & 18 &,$- 0071 \pm .09856$ &, 41816 &,- 1304 &,- 46 &, 80 \\
\hline Size & 18 & $19766,6 \pm 6306,92$ Sq. $\mathrm{m}$ & 26758,02 & 9750 & 300 & 105.000 \\
\hline
\end{tabular}

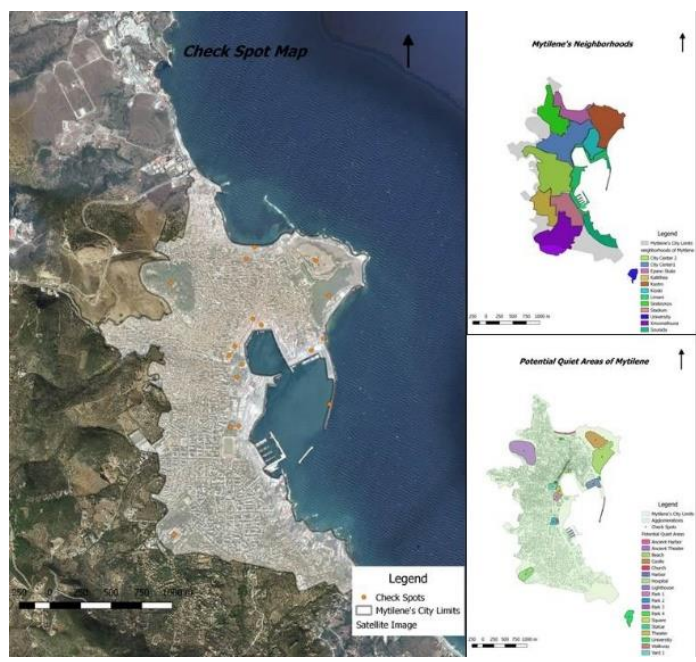

Image 1. Check Spot Map, Mytilene's Neighborhood Map, Mytilene's Potential Quiet Area Map

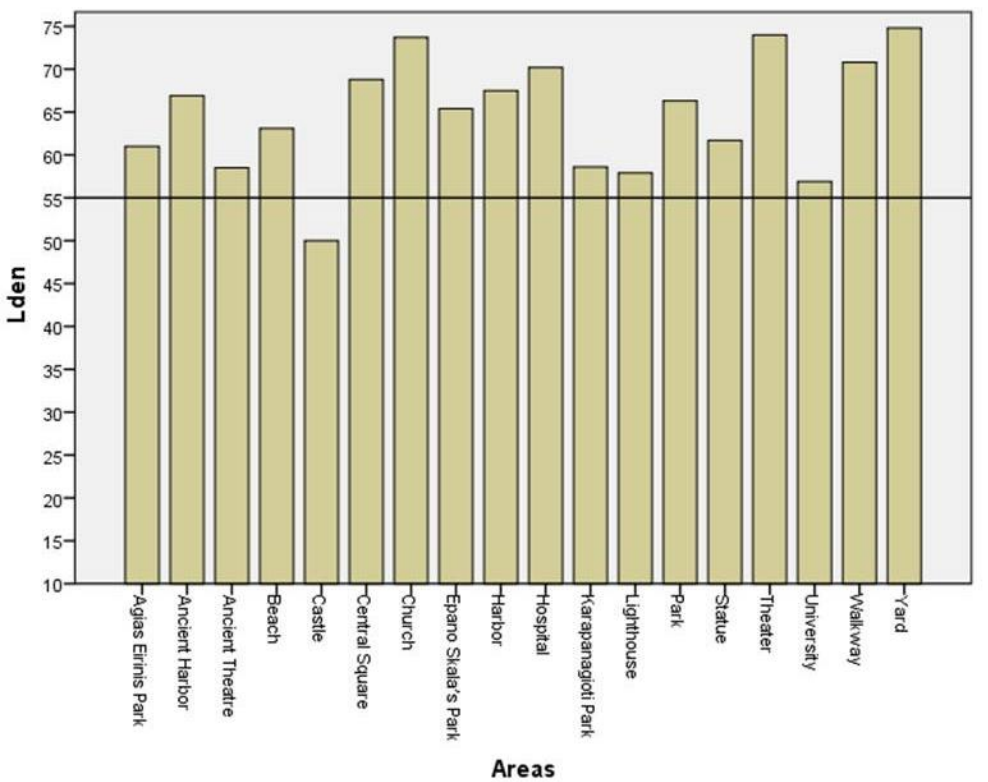

Figure 4. Lden Results Reference line at $55 \mathrm{~dB}(\mathrm{~A})$ representing the Quiet Area Limitation Provided by the EEA (European Environment Agency, 2014) 


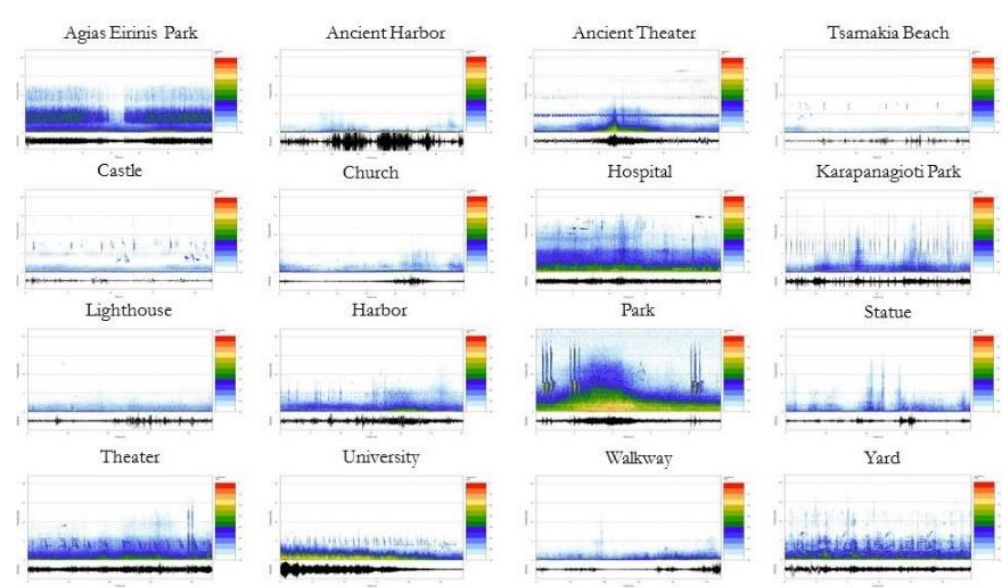

Figure 5. Spectrograms Created Using R Statistics and the Associated R Packages

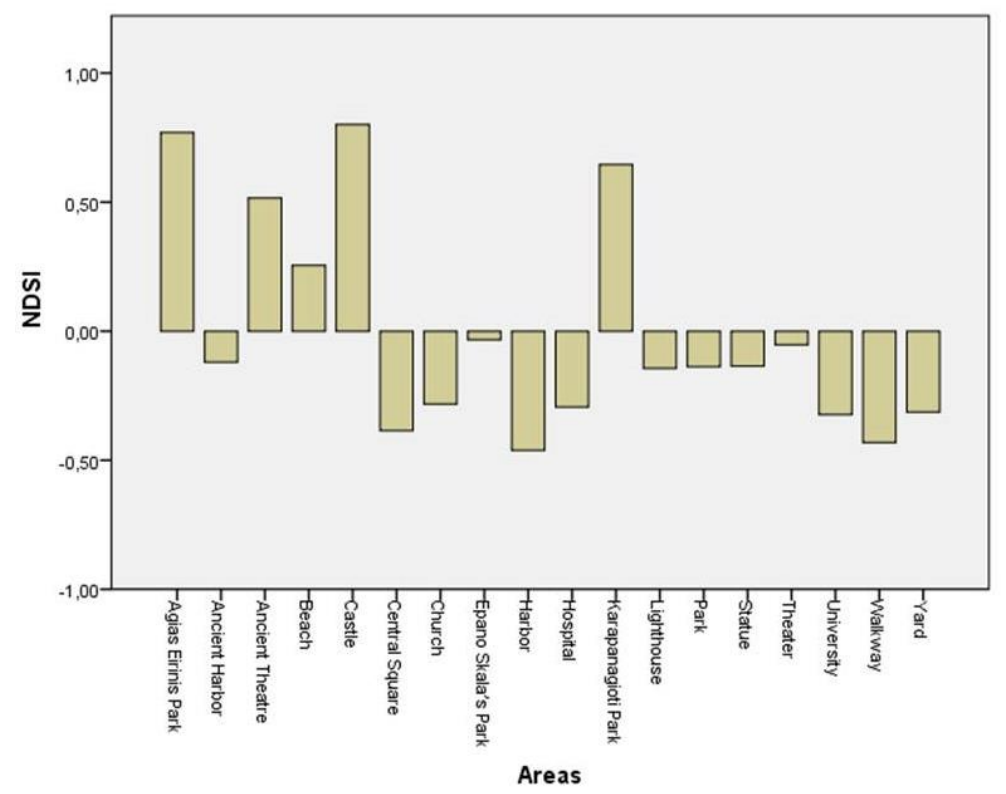

Figure 6. The Resulted Normalized Soundscape Difference Index of each Potential Quiet Area. Values from the areas presented on the diagram's top, consist mainly of biological sounds
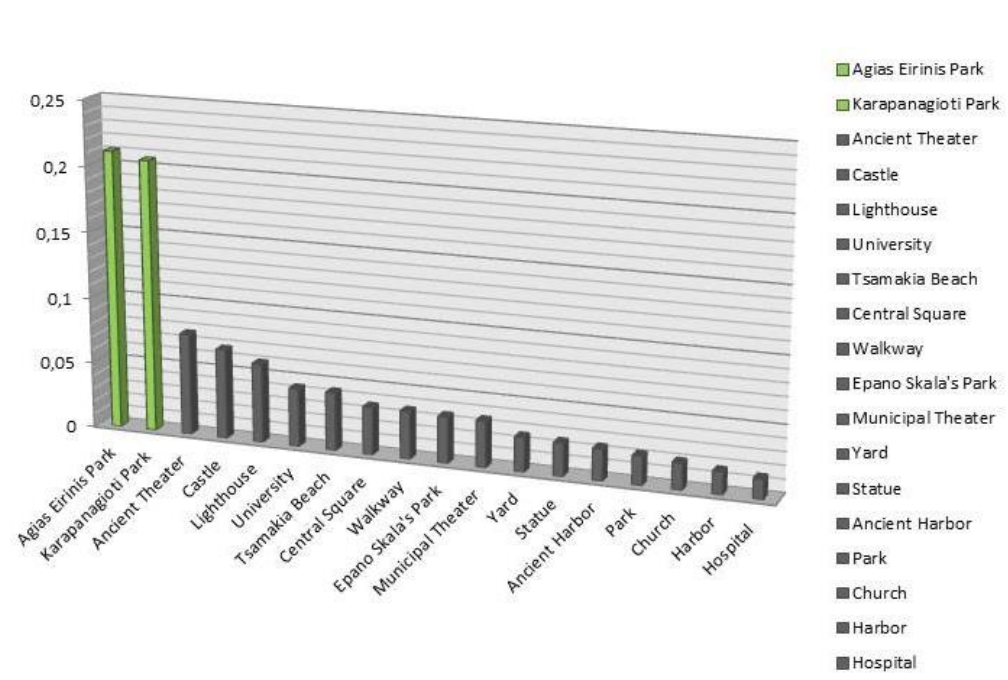

\begin{tabular}{|c|c|}
\hline Area & Weight \\
\hline Agias Eirinis Park & 0,211 \\
\hline Karapanagioti Park & 0,206 \\
\hline Ancient Theater & 0,077 \\
\hline Castle & 0,068 \\
\hline Lighthouse & 0,06 \\
\hline University & 0,044 \\
\hline Tsamakia Beach & 0,044 \\
\hline Central Square & 0,036 \\
\hline Walkway & 0,036 \\
\hline EpanoSkala's Park & 0,035 \\
\hline Municipal Theater & 0,035 \\
\hline Yard & 0,026 \\
\hline Statue & 0,025 \\
\hline Ancient Harbor & 0,024 \\
\hline Park & 0,022 \\
\hline Church & 0,02 \\
\hline Harbor & 0,017 \\
\hline Hospital & 0,015 \\
\hline
\end{tabular}

Figure 7. Analytical Hierarchy Process results. The Agias Eirinis Park and the Karapanagioti Park are Amongst the Top Choices 
The performance matrix that was created in order to assess each check spot in relation with the criteria given by the EEA regarding the identification of quiet areas (table 3). According to the results, the Agias Eirinis Park is the best option for quiet area delimitation due to its high score. The specific park was highlighted as it satisfies almost every criterion set. Amongst the rest check spots, the Karapanagioti Park could be the next best option. Overall,

Table 3. Performance Matrix of Candidate Quiet Areas in Mytilene. The Agias Eirinis Park and the Karapanagioti Park are Amongst the Top Choices

\begin{tabular}{|c|c|c|c|c|c|c|c|c|c|}
\hline Check Spot & $\begin{array}{c}\text { Noise } \\
\text { Indicator }\end{array}$ & NDSI & $\begin{array}{c}\text { Health } \\
\text { Restoration }\end{array}$ & $\begin{array}{c}\text { Nature } \\
\text { Protection }\end{array}$ & Use & Recreation & Visual & Size & $\begin{array}{c}\text { Total } \\
\text { Out } \\
\text { of } 8\end{array}$ \\
\hline $\begin{array}{l}\text { Municipal } \\
\text { Theater }\end{array}$ & 0 & 0 & 0 & 0 & 1 & 0 & 1 & 0 & 2 \\
\hline Walkway & 0 & 0 & 1 & 0 & 1 & 0 & 1 & 0 & 2 \\
\hline Central Square & 0 & 0 & 0 & 0 & 1 & 1 & 1 & 1 & 4 \\
\hline Park & 0 & 0 & 0 & 0 & 0 & 0 & 0 & 1 & 1 \\
\hline School Yard & 0 & 0 & 0 & 0 & 1 & 0 & 0 & 0 & 1 \\
\hline Church & 0 & & 1 & 0 & 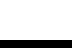 & 0 & 1 & . & 2 \\
\hline Agias Eirinis Park & 0 & 1 & 0 & 1 & 1 & 1 & 1 & 1 & 6 \\
\hline $\begin{array}{l}\text { Karapanagioti } \\
\text { Park }\end{array}$ & 0 & 1 & 0 & 1 & 1 & 1 & 1 & 1 & 6 \\
\hline Ancient Theatre & 0 & 1 & 0 & 1 & 0 & 0 & 0 & 0 & 1 \\
\hline Ancient Harbor & 0 & 0 & 0 & 0 & 0 & 0 & 1 & 0 & 1 \\
\hline E. Skala's Park & 0 & 0 & 0 & 0 & 1 & 1 & 0 & 1 & 3 \\
\hline Castle & 1 & 1 & 1 & 1 & 0 & 0 & 1 & 0 & 5 \\
\hline Statue of Liberty & 0 & 0 & 1 & 0 & 0 & 0 & 1 & 0 & 2 \\
\hline Tsamakia beach & 0 & 1 & 0 & 1 & 0 & 1 & 1 & 0 & 4 \\
\hline Harbor & 0 & 0 & 0 & 0 & 0 & 0 & 0 & 0 & 0 \\
\hline Lighthouse & 0 & 0 & 1 & 0 & 1 & 0 & 1 & 0 & 3 \\
\hline Hospital & 0 & 0 & 0 & 0 & 1 & 0 & 0 & 0 & 1 \\
\hline University & 1 & 0 & 0 & 0 & 1 & 0 & 0 & 0 & 2 \\
\hline
\end{tabular}

Similarly to the performance matrix results, Mytilene's green urban spaces Agias Eirinis Park (39.102933, 26.555071) and Karapanagioti Park (39.099405, 26.554469) located in the city's center are the best available options (image 2). The results demonstrate a priority to urban green areas, regarding their acoustic profile and other characteristics similar to recreation.

The resulted candidate quiet areas were the "Agias Eirinis Park" and the Karapanagioti Park; therefore two soundwalks were specifically designed (image 3 ) in order to assess the acoustic perception of individuals. The soundwalk conducted practically involved city residents, in order to address environmental noise. The participant's acoustic preferences compose a new more agreeable soundscape and therefore reshape the landscape. Furthermore, the information on what could be heard during the soundwalk could be used in future research in order to detect acoustic differences that may occur in time (table 4, table 5).
Mytilene is characterized by a plethora of cultural and natural values, while recreation seems to be a very important aspect of the city.

By evaluating the alternative areas using the Analytical Hierarchy Process (AHP) Priority Estimation Tool (PriEsT), the weights of each alternative was calculated (Fig. 7). 


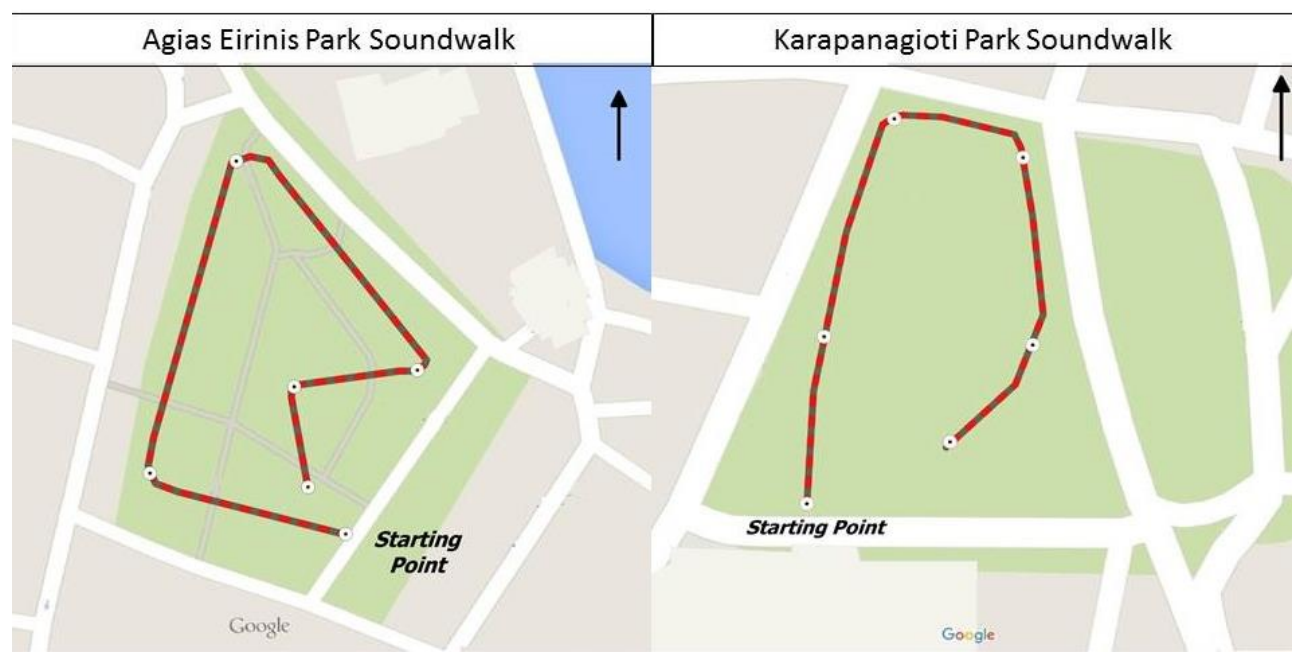

Image 3. Agias Eirinis Park and Karapanagioti's Park Soundwalk Routes, Source: Google Maps

Table 4. Agias Eirinis Park Soundwalk Results

\begin{tabular}{|c|c|c|c|c|c|}
\hline \multicolumn{6}{|c|}{ City Park “Agias Eirinis” Soundwalk } \\
\hline & Stop 1 & Stop 2 & Stop 3 & Stop 4 & Stop 5 \\
\hline 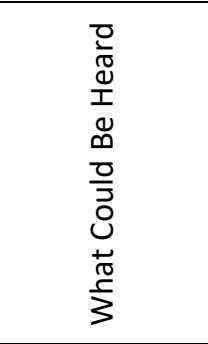 & $\begin{array}{l}\text { Car Engine } \\
\text { Swing Squeak } \\
\text { Children } \\
\text { Airplane } \\
\text { People Talking } \\
\text { Birds }\end{array}$ & $\begin{array}{c}\text { Car Engine } \\
\text { People Talking } \\
\text { Birds } \\
\text { Rustling Leaves } \\
\text { Footsteps } \\
\text { Insects }\end{array}$ & $\begin{array}{c}\text { Car Engine } \\
\text { Footsteps } \\
\text { People Talking } \\
\text { Birds } \\
\text { Motorbike } \\
\text { Wind Brewing }\end{array}$ & $\begin{array}{c}\text { Car Engine } \\
\text { Children } \\
\text { People Talking } \\
\text { Wind Brewing } \\
\text { Birds } \\
\text { Footsteps }\end{array}$ & $\begin{array}{l}\text { Car Engine } \\
\text { Children } \\
\text { Laughter } \\
\text { Birds } \\
\text { Motorbike } \\
\text { Footsteps } \\
\text { Vehicle Horns } \\
\text { Wind Brewing }\end{array}$ \\
\hline Land Use & Church & Resting Area & Entrance & Playground & Park Center/Cafe \\
\hline $\begin{array}{l}\text { Dominant } \\
\text { Sound }\end{array}$ & Anthrophony & Anthrophony & Anthrophony & Biophony & Biophony \\
\hline $\begin{array}{c}\text { Preferable } \\
\text { Sound }\end{array}$ & Birds & Water Sounds & Birds & Music & Water Sounds \\
\hline $\begin{array}{c}\text { Measured Leq } \\
\mathrm{dB}(\mathrm{A}) \\
\end{array}$ & 63,6 & 60,9 & 64,3 & 61,4 & 63.7 \\
\hline
\end{tabular}

Table 5. Karapanagioti Park Soundwalk Results

\begin{tabular}{|c|c|c|c|c|c|}
\hline \multicolumn{6}{|c|}{ Karapanagioti Soundwalk } \\
\hline & Stop 1 & Stop 2 & Stop 3 & Stop 4 & Stop 5 \\
\hline 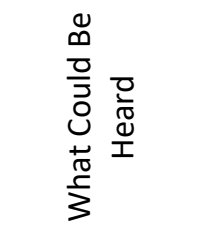 & $\begin{array}{l}\text { Car Engine } \\
\text { People Talking } \\
\text { Birds }\end{array}$ & $\begin{array}{c}\text { Car Engine } \\
\text { Birds } \\
\text { Rustling Leaves } \\
\text { Footsteps }\end{array}$ & $\begin{array}{c}\text { Car Engine } \\
\text { Footsteps } \\
\text { People Talking } \\
\text { Birds } \\
\text { Motorbike }\end{array}$ & $\begin{array}{c}\text { Car Engine } \\
\text { People Talking } \\
\text { Wind Brewing } \\
\text { Birds } \\
\text { Insects }\end{array}$ & $\begin{array}{c}\text { Car Engine } \\
\text { Nature Sounds } \\
\text { Birds } \\
\text { People Talking }\end{array}$ \\
\hline Land Use & Entrance 1 & $\begin{array}{c}\text { Resting Area/ } \\
\text { Foliage }\end{array}$ & Entrance 2 & Cafeteria & Foliage \\
\hline $\begin{array}{c}\text { Dominant } \\
\text { Sound }\end{array}$ & Anthropophony & Anthropophony & Anthropophony & Anthropophony & Biophony \\
\hline $\begin{array}{c}\text { Preferable } \\
\text { Sound }\end{array}$ & Water Sounds & Nature Sounds & Music & Birds & Water Sounds \\
\hline $\begin{array}{c}\text { Measured Leq } \\
\mathrm{dB}(\mathrm{A})\end{array}$ & 54,8 & 63,4 & 60,6 & 52,8 & 55,4 \\
\hline
\end{tabular}

The presence of road traffic noise was in all cases noticeable. From the responses given by the soundwalkers on the quality of noise at each stop, it resulted that the least favorite noises were dominant, but less diverse. It is 
obvious that vital auditory information about an area's soundscape could derive through careful listening.

\section{Conclusions}

The necessary steps that must be taken, to preserve and protect quiet areas, do not concern these areas alone, but mainly, the areas surrounding them. Once a quiet area is delimited by the member state, it is of obvious importance the proper design and promotion of peace outside this area in order to create a zone of protection from environmental noise. "Quietness" in an area could be achieved not only by controlling noise emissions but also by enhancing positive sounds. In order to improve the area selection procedure, information regarding the ecology of each area should be included. Biological and geophysical sounds are directly associated with the ecological aspects of a candidate quiet area. Vegetation and bird species should be identified, in order to highlight the positive effects they might have on the overall soundscape (Irvine, 2009). Furthermore, research on other forms of life (e.g. insects) on each area under consideration could contribute to the classification of the biodiversity levels and therefore properly assess nature protection.

The goal of this paper was to create an easy to use and cost effective quiet area identification procedure. Nevertheless, "quietness", as a concept still remains vague and further research is needed in order to conclude whether it is an attribute that could be created or simply and preferably, preserved.

\section{References}

Aspuru I., Garcia I., Bartalucci C., Borchi F., Carfagni M., Governi L., Bellomini R., Luzzi S., Wolfert H. and Gaudibert P. (2016), LIFE+2010 QUADMAP Project: a new methodology to select, analyze and manage Quiet Urban Areas defined by the European Directive 2002/49/EC, Noise Mapp., 3, 120-129.

Booi H. and Van Den Berg F. (2012), Quiet Areas and the need for quietness in Amsterdam, Environ. Research and Public Health, 9, 1030-1050.

Brambilla G., Gallo V., Asdrubali F. and D'Alessandro F. (2013), The perceived quality of soundscape in three urban parks in Rome, Acoustical Society of America, 134, 832 - 839.

Brown L. and Lam K.C. (1987), Urban Noise Surveys, Applied Acoustics, 20, 23-39.

Bruce N.S. and Davies W.J. (2014), The effects of expectation on the perception of soundscapes, Applied Acoustics, 85, 1-11.

Cohn J.P. (2008), Citizen Science: Can Volunteers Do Real Research?, BioScience, 58, 192-197.

Conrad C.C. and Hilchey K.G. (2011), A review of citizen science and community-based environmental monitoring: issues and opportunities, Environmental Monitoring and Assessment, 176, 273-291.

Davies W.J., Adams M.D., Bruce N.S., Cain R., Carlyle A., Cusack P., Hall D.A., Hume K.I., Irwin A., Jennings P., Marselle M., Plack C.J. and Poxon J. (2013), Perception of soundscapes: An interdisciplinary approach, Applied Acoustics, 74, 224-231.

European Environment Agency, Expert Panel on Noise, Good Practice Guide on Quiet Areas, EEA Technical report No 4, 2014, ISSN 1725-2237, DOI: 10.2800/12611
European Union (EU). Directive 2002/49/EC relating to the assessment and management of environmental noise. Official journal of the European communities, No. L. 189; 2002

Farina A. and Pieretti N. (2012), The soundscape ecology: A new frontier of landscape research and its application to islands and coastal systems, Journal of Marine and Island Cultures, 1, 21-26.

Fisher B., Turner R.K. and Morling P. (2009), "Defining and classifying ecosystem services for decision making", Ecological Economics, 68, 643-653

Friedmann J. (2010), Place and Place-Making in Cities: A Global Perspective, Planning Theory and Practice, 11, 149-165.

Fuller S., Axel A.C., Tucker D. and Gage S.H. (2015), Connecting soundscape to landscape: Which acoustic index best describes landscape configuration?, Ecological Indicators, 58, 207-215.

Gidlöf-Gunnarsson A. and Öhrström E. (2007), Noise and wellbeing in urban residential environments: The potential role of perceived availability to nearby green areas, Landscape and Urban Planning, 83, 115-126.

Hellenic Statistical Authority, EI.STAT., Statistical Themes, Population, Mytilene Lesvos, http://www.statistics.gr/portal/page/portal/ESYE/PAGEthemes?p_param=A1603, Last accessed in 9/10/2015

Irvine K.N., Devine-Wright P., Payne S.R., Fuller R.A., Painter B. and Gaston K.J. (2009), Green space, soundscape and urban sustainability: an interdisciplinary, empirical study, Local Environment: The International Journal of Justice and Sustainability, 14, 155-172.

Jennings P. and Cain R. (2013), A framework for improving urban soundscapes, Applied Acoustics, 74, 293-299.

Jeon J.Y., Hong J.Y. and Le P.J. (2013), Soundwalk approach to identify urban soundscapes individually, Acoustical Society of America, 134, 803-812.

Jeon J.Y. and Hong J.Y. (2015), Classification of urban park soundscapes through perceptions of the acoustical environments, Landscape and Urban Planning, 141, 100-111.

Kasten E.P., Gage S.H., Fox J. and Joo W. (2012), The remote environmental assessment laboratory's acoustic library: An archive for studying soundscape ecology, Ecological Informatics, 12, 50-67.

Ligges U., Krey S., Mersmann O. and Schnackenberg S. (2013,. tuneR: Analysis of music, http://r-forge.rproject.org/projects/tuner/

Loss S.R., Loss S.S., Will T. and Marra P.P. (2015), Linking placebased citizen science with large-scale conservation research: A case study of bird-building collisions and the role of professional scientists, Biological Conservation, 184, 439-445.

Luymes D.T. and Tamminga K. (1995), Integrating public safety and use into planning urban greenways, Landscape and Urban Planning, 33, 391-400.

Matsinos Y.G, Mazaris A.D., Papadimitriou K.D., Mniestris A., Hatzigiannidis G., Maioglou D. and Pantis J.D. (2008), Spatiotemporal variability in human and natural sounds in a rural landscape, Landscape Ecology, 23, 945-959.

Munang R. T., Thiaw I. and Rivington M. (2011), Ecosystem Management: Tomorrow's Approach to Enhancing Food Security under a Changing Climate, Sustainability, 3, 937-954

R Core Team (2015). R: A language and environment for statistical computing. R Foundation for Statistical Computing, Vienna, Austria, http://www.R-project.org/ 
Resnik D.B., Elliott K.C. and Miller A.K. (2015), A framework for addressing ethical issues in citizen science, Environmental Science and Policy, 54, 475-481.

Saaty T.L. (2004), Decision Making - The Analytic hierarchy and Network Processes (AHP/ANP), Journal of Systems and Science and Systems Engineering, 13, 1-35.

Saaty T.L. (2008), Relative measurement and its generalization in decision making why pairwise comparisons are central in mathematics for the measurement of intangible factors the analytic hierarchy/network process, Revista de la Real Academia de Ciencias Exactas, Fisicas y Naturales. Serie A. Matematicas, 102, 251-318

Sharifi A. (2015), From Garden City to Eco-urbanism: The quest for sustainable neighborhood development, Sustainable Cities and Society, 20, 1-16.

Silvertown J. (2009), A new dawn for citizen science, Trends in Ecology and Evolution, 24, 467-471.

Siraj S., Mikhailovc L. and Keaned J.A. (2015), PriEsT: An Interactive Decision Support Tool to Estimate Priorities from Pairwise Comparison Judgments, International Transactions in Operational Research, 22, 217-235

Smith M.E. (2010), The archaeological study of neighborhoods and districts in ancient cities, Journal of Anthropological Archaeology, 29(2), 137-154.

Steele K., Carmel Y., Cross J. and Wilcox C. (2009), Uses and Misuses of Multicriteria Decision Analysis (MCDA) in Environmental Decision Making, Risk Analysis, 29, 26-33.

Sueur J., Aubin T. and Simonis C. (2008), Seewave: a free modular tool for sound analysis and synthesis, Bioacoustics, 18, 213-226.

Tratalos J., Fuller R.A., Warren P.H., Davies R.G. and Gaston K.J. (2007), Urban form, biodiversity potential and ecosystem services, Landscape and Urban Planning, 83, 308-317.

Villanueva-Rivera L.J., Pijanowski B.C., Doucette J. and Pekin B.(2011), A primer of acoustic analysis for landscape ecologists, Landscape Ecology, 26, 1233-1246

Votsi N.E.P., Drakou E.G., Mazaris A.D., Kallimanis A.S. and Pantis J.D., Distance-based assessment of open country Quiet Areas in Greece, Landscape and Urban Planning, 104, 279-288.

Whitelaw G., Vaughan H., Craig B. and Atkinson D. (2003), Establishing the Canadian Community Monitoring Network, Environmental Monitoring and Assessment, 88, 409-418.

WHO, Guidelines for Community Noise, Geneva, 1999, http://www.who.int/docstore/peh/noise/guidelines2.html, Last accessed in 8/9/2015

Yang L., Zhang L., Li Y. and Wu S.(2015), Water-related ecosystem services provided by urban green space: A case study in Yixing City (China), Landscape and Urban Planning, 136, 40-51.

Zeileis A. (2014), ineq: Measuring Inequality, Concentration, and Poverty, $\mathrm{R}$ package version 0.2-13, http://CRAN.Rproject.org/package=ineq 